Прилози, Одд. мат. тех. науки, МАНУ, XXVII-XXVIII, 1-2 (2006-2007), стр. 55-66 Contributions, Sec. Math. Tech. Sci., MANU, XXVII-XXVIII, 1-2 (2006-2007), pp. 55-66

ISSN 0351-3246

UDC: 517.518 .389

\title{
QUASI-PERIODIC SOLUTIONS TO THE RICCATI DIFFERENTIAL EQUATION
}

\author{
Marija Kujumdžieva-Nikoloska, Jordanka Mitevska
}

A b s t r a c t: In this paper we give some conditions for existence of quasi-periodic solutions to the Riccati differential equation and find this solution in a case of a constant quasi-period.

Key words with abbreviation: differential equation (DE); quasiperiod (QP); quasi-periodic solution (QPS); quasi-periodic coefficient (QPC); equation (eq.)

\section{PRELIMINARY}

Let the Riccati differential equation

$$
y^{\prime}(x)+f(x) y(x)+g(x) y^{2}(x)+h(x)=0 \quad(g(x) \neq 0)
$$

be given. We want to find QPS $y=y(x)$ for (1), i.e. to find the solution that satisfies the relation

$$
y(x+\omega)=\lambda(x, \omega(x)) y(x)=\lambda(x) y(x), x, x+\omega \in D_{y}
$$

where $\omega=\omega(x)$ is QP and $\lambda=\lambda(x)$ is QPC for the function $y=y(x)$.

The following theorem holds.

Theorem 1.1. If DE (1) has QPS $y=y(x)$ with QP $\omega=\omega(x)$ and QPC $\lambda(x)$, then it is reduced to the algebraic equation with respect to the QPS $y$ 


$$
\begin{gathered}
\left(-\frac{1}{t^{\prime}} \lambda(x) g(x)+\lambda^{2}(x) g(t)\right) y^{2}(x)+\left(\frac{\lambda^{\prime}(x)}{t^{\prime}}-\frac{\lambda(x)}{t^{\prime}} f(x)+\lambda(x) f(t)\right) y(x)+ \\
+\left(-\frac{\lambda(x)}{t^{\prime}} h(x)+h(t)\right)=0, t=x+\omega(x)
\end{gathered}
$$

Proof. Under the conditions of the theorem we have the system

$$
\left.\begin{array}{l}
y^{\prime}(x)+f(x) y(x)+g(x) y^{2}(x)+h(x)=0 \\
y^{\prime}(t)+f(t) y(t)+g(t) y^{2}(t)+h(t)_{\mid t=x+\omega}=0 \\
y(t)=\lambda(x) y(x) \\
\frac{d}{d x} y(t)=\lambda^{\prime}(x) y(x)+\lambda(x) y^{\prime}(x)
\end{array}\right\}
$$

from where

$$
y^{\prime}(x)=-f(x) y(x)-g(x) y^{2}(x)-h(x)
$$

and

$$
y^{\prime}(t)=\frac{1}{t^{\prime}}\left(\lambda^{\prime}(x) y(x)+\lambda(x) y^{\prime}(x)\right) .
$$

Subtituting (5) and (6) in the second equation of the system (4), after short transfomations, we obtain (3).

Remark 1.1. In general, solving equation (3) is not a simple problem and we can solve it only in some special cases. So, here we consider QPS for (1) with a constant QP and a constant QPC.

\section{QUASIPERIODIC SOLUTIONS WITH CONSTANT QUASI-PEROD AND CONSTANT QUASIPERIODIC COEFFICIENT}

Theorem 2.1. Let DE (1) have QPS $y=y(x)$ with a constant QP $\omega=\boldsymbol{c}$ and a constant QPC $\lambda>\mathbf{0}$. Then it is reduced, with respect to QPS $y$, to the equation: 


$$
\lambda(-g(x)+\lambda g(t)) y^{2}(x)+\lambda(-f(x)+f(t)) y(x)+(-\lambda h(x)+h(t))=0 /_{t=x+c}
$$

Proof. Substituting in eq. (3) $\omega=c, \omega^{\prime}-0, t=x+c, t^{\prime}=1$ we obtain eq. (7).

Theorem 2.2. Let

$1^{\circ}$ DF (1) have QPS $y_{1}(x)$ with a constant QP $\omega=\boldsymbol{c}$ and a constant QPC $\lambda>0$;

$2^{0}$ the coefficients $f(x), g(x), h(x)$ are QPF with the same QP $\omega=\boldsymbol{c}$ and QPC $\lambda_{1}=1, \lambda_{2}=\frac{1}{\lambda}, \lambda_{3}=\lambda$ respectively;

$3^{0}$ the general solution for the equation

$$
z^{\prime}-\left(f(x)+2 g(x) y_{1}(x)\right) z-g(x)=0
$$

is QPF with QP $\omega=c$ and $\mathrm{QPC} \frac{1}{\lambda}$.

Then every solution for DE (1) is QPF with QP $\omega=c$ and QPC $\lambda>\mathbf{0}$.

Proof. Under the given conitions follows that the general solutions for DE (1) is $y(x)=y_{1}(x)+\frac{1}{z(x)}$, where $z=z(x)$ [1] is QPS for the eq. (8). Since the coefficients $f(x), g(x), h(x)$ satisfy the relations $y(t)=f(x), g(t)=\frac{1}{\lambda} g(x)$, $h(i)=\lambda h(x) /_{t=x+c}$, we get

$$
y(t)=y_{1}(t)+\frac{1}{z(t)}=\lambda\left(y_{1}(x)+\frac{1}{z(x)}\right)=\lambda y(x),
$$

and

$$
y^{\prime}(t)+f(t) y(t)+g(t) y^{2}(t)+h(t)_{/ t=x+c}=
$$




$$
\begin{aligned}
& =\lambda y^{\prime}(x)+f(x) \cdot \lambda y(x)+\frac{1}{\lambda} g(x) \cdot \lambda^{2} y^{2}(x)+\lambda h(x)= \\
& =\lambda\left(y^{\prime}(x)+f(x) y(x)+g(x) y^{2}(x)+h(x)\right)=\lambda \cdot 0=0 .
\end{aligned}
$$

Remark 2.1 If DE (1) has QPS $y_{1}(x)$ then its general solution is

$$
y(x)=y_{1}(x)+\frac{1}{z(x)}=y_{1}(x)+\frac{1}{C a(x)+b(x)},
$$

where $a(x)=e^{\int\left(f(x)+2 g(x) y_{1}(x)\right) d x}, \quad b(x)=a(x) \int \frac{g(x)}{a(x)} d x$.

\section{Example 2.1. Let}

$$
y^{\prime}+(3+\operatorname{ctg} x) y-\frac{e^{x}}{\sin x} y^{2}-e^{-x}(\sin x+2 \cos x)=0 .
$$

This equation has a particular solution $y_{1}=e^{-x} \sin x$ that is QPF with a QP $\omega=2 \pi$ and QPC $\lambda=e^{-2 \pi}$. Since the coefficients $f(x), g(x), h(x)$ are a QPF with the same QP $\omega=2 \pi$ and QPC $1, e^{2 \pi}=\frac{1}{\lambda}, e^{-2 \pi}$ respectively, according to the Theorem 2.2. every solution for the given equation is QPF. Indeed, its general solution is $y=e^{-x}\left(\sin x+\frac{1}{C \sin x+\cos x}\right)$, that is QPF with QP $\omega=2 \pi$ and QPC $\lambda=e^{-2 \pi}$.

\section{Theorem 2.3. If}

$1^{\circ} \mathrm{DE}(1)$ has two QPS $y_{1}(x)$ and $y_{2}(x)$, with QP $\omega=c$ and QPC $\lambda>0$,

$2^{0}$ the coefficients $f(x), g(x), h(x)$ in DE (1) are QPF with QP $\omega=c$ and QPC $\lambda_{1}=1, \lambda_{2}=\frac{1}{\lambda}, \lambda_{3}=\lambda$ respectively, 


$$
3^{\mathrm{o}} \text { for } \varphi(x)=e^{-\int g(x)\left(y_{1}(x)-y_{2}(x)\right) d x}, z(x)=\frac{C \varphi(x)-1}{C \varphi(x)} \frac{1}{y_{2}(x)-y_{1}(x)} \text { is }
$$

a general QPS for (8) with QP $\omega=c$ and QPC $\frac{1}{\lambda}$,

then every solution for the DE (1) is QPF with QP $\omega=c$ and QPC $\lambda$.

Proof. It can be proved in a similar manner as the previous theorem.

Remark 2.2. If the DE (1) has two QPS $y_{1}=y_{1}(x)$ and $y_{2}=y_{2}(x)$ with QP $\omega=c$ and QPC $\lambda>0$, then its general solution is

$$
\begin{aligned}
y(x)=y_{1}(x)+\frac{1}{z(x)} & =\frac{1}{1-C \phi(x)} y_{1}(x)+\left(1-\frac{1}{1-C \phi(x)}\right) y_{2}(x)= \\
& =\mu(x, C) y_{1}(x)+(1-\mu(x, C)) y_{2}(x),
\end{aligned}
$$

i.e. if the solution is in the form $y(x)=y_{1}(x)+\frac{1}{C_{1} a(x)+b(x)}$, then

$$
a(x)=\frac{-1}{\varphi(x)\left(y_{1}(x)-y_{2}(x)\right)}, b(x)=\frac{-1}{y_{1}(x)-y_{2}(x)} .
$$

Example 2.2. Let

$$
y^{\prime}+\frac{2 \cos ^{2} x-2 \cos x-1}{\sin x-\cos x} y+e^{x} y^{2}+e^{-x}\left(\sin x \cos x+\frac{1}{\sin x-\cos x}\right)=0 .
$$

This equation has particular solutions $y_{1}=e^{-x} \sin x$ and $y_{2}(x)=e^{-x} \cos x$ that are QPF with QP $\omega=2 \pi$ and QPC $\lambda=e^{-2 \pi}$. Since the coefficients $f(x)$, $g(x), h(x)$ are QPF with the same QP $\omega=2 \pi$ and QPC $1, e^{2 \pi}=\frac{1}{\lambda}$, $e^{-2 \pi}=\lambda$ respectively, according to the Theorem 2.3. every solution is QPF. 
Indeed, the general solution is $y=e^{-x}\left(\sin x+\frac{e^{\sin x+\cos x}(\cos x-\sin x)}{C_{1}+e^{\sin x+\cos x}}\right)$, that is QPF with QP $\omega=2 \pi$ and QPC $\lambda=e^{-2 \pi}$.

\section{Theorem 2.4. If}

$1^{\circ} \mathrm{DE}(1)$ has QPS $y_{1}(x), y_{2}(x)$ and $y_{3}(x)$ with QP $\omega=c$ and QPC $\lambda>0$,

$2^{\circ}$ the coefficients $f(x), g(x), h(x)$ in DE (1) are QPF with QP $\omega=c$ and QPC $\lambda_{1}=1, \lambda_{2}=\frac{1}{\lambda}, \lambda_{3}=\lambda$ respectively,

$$
3^{\mathrm{o}} \text { for } \psi(x)=\frac{y_{3}(x)-y_{1}(x)}{y_{3}(x)-y_{2}(x)}, z(x)=\frac{k \psi(x)-1}{k \psi(x)} \frac{1}{y_{2}(x)-y_{1}(x)} \text { is a gene- }
$$

ral QPS with QP $\omega=c$ and QPC $\frac{1}{\lambda}$ to the equation (8),

then every solution for DE (1) is QPF with QP $\omega=c$ and QPC $\lambda$.

Proof. It can be proved in a similar manner as the Theorem 2.2.

Remark 2.3 The general solution for DE (1) is

$$
\begin{gathered}
y(x)=y_{1}(x)+\frac{1}{z(x)}=\frac{1}{1-k \psi(x)} y_{1}(x)+\left(1-\frac{1}{1-k \psi(x)}\right) y_{2}(x)= \\
=v(x, k) y_{1}(x)+(1-v(x, k)) y_{2}(x)
\end{gathered}
$$

i.e. if $y(x)=y_{1}(x)+\frac{1}{C_{2} a(x)+b(x)}$, then $a(x)=\frac{-1}{\psi(x)\left(y_{1}(x)-y_{2}(x)\right)}$,

$$
b(x)=\frac{-1}{y_{1}(x)-y_{2}(x)} .
$$


Example 2.3. Let

$$
\begin{gathered}
y^{\prime}+\frac{1-\sin x+\cos x+2 \sin ^{2} x-2 \sin ^{3} x}{(1-\sin x)(1-\cos x)(\cos x-\sin x)} y+e^{x} \frac{1-\sin x-\cos x}{(1-\sin x)(1-\cos x)(\cos x-\sin x)} y^{2}+ \\
+e^{-x} \frac{-1-\sin x-\sin x \cos x-\sin ^{2} x \cos x+\sin ^{3} x}{(1-\sin x)(1-\cos x)(\cos x-\sin x)} y=0 .
\end{gathered}
$$

This equation has particular solutions $y_{1}=e^{-x} \sin x, y_{2}(x)=e^{-x} \cos x$ and $y_{3}(x)=e^{-x}$. They are QPF with QP $\omega=2 \pi$ and QPC $\lambda=e^{-2 \pi}$. Since the coefficients $f(x), g(x), h(x)$ are QPF with the same QP $\omega=2 \pi$ and QPC 1, $e^{2 \pi}=\frac{1}{\lambda}, e^{-2 \pi}=\lambda$ respectively, according to the Theorem 2.4. every solution is QPF. Indeed, the general solution is

$$
y=e^{-x}\left(\sin x+\frac{\cos x-\sin x-\sin x \cos x+\sin ^{2} x}{C(1-\cos x)+(1-\sin x)}\right),
$$

that is QPF with QP $\omega=2 \pi$ and QPC $\lambda=e^{-2 \pi}$.

Theorem 2.5. Let the DE (1) have one QPS with QP $\omega=c$ and QPC $\lambda=\frac{\lambda_{3}}{\lambda_{1}}$, and the coefficients $f(x) \neq 0, g(x), h(x)$ in DE (1) be QPF with QP $\omega=c$ and QPC $\lambda_{1} \neq 1, \lambda_{2}=\frac{1}{\lambda}, \lambda_{3}$, respectively. Then the QPS for DE (1) is

$$
y=-\frac{h(x)}{f(x)}
$$

if the relation

$$
\left(\frac{h(x)}{f(x)}\right)^{\prime}-g(x)\left(\frac{h(x)}{f(x)}\right)^{2}=0
$$

is satisfied.

Прилози, Одд. мат. тех. науки, XXVII-XXVIII, 1-2 (2006-2007), стр. 55-66 
Proof. Under the conditions, $f(x+c)=\lambda_{1} f(x), \quad g(x+c)=\frac{1}{\lambda} g(x)$, $h(x+c)=\lambda_{3} h(x)$, QPS to the DE (1) is also QPS to the equation

$$
\left(\lambda_{1}-1\right) f(x) y=-\frac{1}{\lambda}\left(\lambda_{3}-\lambda\right) h(x),
$$

from which we get

$$
y=\mu_{1} \cdot \frac{h(x)}{f(x)}
$$

where $\mu_{1}=-\frac{\lambda_{3}-\lambda}{\lambda\left(\lambda_{1}-1\right)}$. Solution (11) is QPF with QP $\omega=c$ and QPC $\lambda=\frac{\lambda_{3}}{\lambda_{1}}$ for which $\mu_{1}=-1$. Thus, from (11) we obtain (9). So, since the solution (9) is also the solution to DE (1), we obtain that the coefficients $f, g, h$ have to satisfy the relation (10).

Corollary 2.1. Under the conditions of the Theorem 2.5. QPS for DE (1) is given by

$$
y=\frac{1}{\int_{x_{0}}^{x} g(x) d x+C\left(x_{0}\right)}, C\left(x_{0}\right)=-G\left(x_{0}\right), G^{\prime}(x)=g(x) .
$$

Proof. From the relation (10) we have

$$
\frac{h(x)}{f(x)}=-\frac{1}{\int_{x_{0}}^{x} g(x) d x+C\left(x_{0}\right)} .
$$

Since, under he conditions of the Theorem 2.5., QPS for DE (1) is $y=-\frac{h(x)}{f(x)}$, we obtain (12). 
Example 2.4. The Riccati equation

$$
y^{\prime}-e^{-x+\sin x} \cos x \cdot y+e^{-2 x+\sin x}(-2+\cos x) \cdot y^{2}+e^{x} \cos x=0
$$

has coefficients $f(x)=-e^{-x+\sin x} \cdot \cos x, \quad g(x)=e^{-2 x+\sin x} \cdot(-2+\cos x)$, $h(x)=e^{x} \cdot \cos x$, which are QPF with the same QP $\omega=2 \pi$ and QPC $\lambda_{1}=e^{-2 \pi}$, $\lambda_{2}=e^{-4 \pi}, \lambda_{3}=e^{2 \pi}$, respectively, and satisfy the condition (10). Thus, according to the Theorem 2.5, QPS for the given DE is $y=-\frac{h(x)}{f(x)}=e^{2 x-\sin x}(\omega=2 \pi$, $\left.\lambda=e^{4 \pi}=\frac{\lambda_{3}}{\lambda_{1}}\right)$, or using (12):

$$
y=-\frac{1}{\int_{x_{0}}^{x} g(x) d x+C_{0}}=-\frac{1}{\int_{x_{0}}^{x} e^{-2 x+\sin x}(-2+\cos x) d x+C_{0}}=e^{2 x-\sin x} .
$$

Theorem 2.6. Let the coefficients $f(x), g(x), h(x)$ in DE (1) be QPF with QP $\omega=c$ and QPC $\lambda_{1} \neq 1, \lambda_{2} \neq \frac{1}{\lambda}, \lambda_{3}$ respectively.

1) If DE (1) has QPS $y=y(x)$ with QP $\omega=c$, QPC $\lambda \neq \frac{\lambda_{1}}{\lambda_{2}}$ and $f(x) \neq 0$, then $y=0$.

2) If DE (1) has QPS $y=y(x)$ with QP $\omega=c$, QPC $\lambda \neq \frac{\lambda_{1}}{\lambda_{2}}$ and $f(x)=0$, then $y=0$.

3) If DE (1) has QPS $y=y(x)$ with QP $\omega=c$, QPC $\lambda=\frac{\lambda_{1}}{\lambda_{2}}$ and $h(x)=0$, then $y=0$ or $y=C$ when $C g(x)+f(x)=0$, 
4) If $\mathrm{DE}(1)$ has QPS $y=y(x)$ with QP $\omega=c$, QPC $\lambda=\frac{\lambda_{1}}{\lambda_{2}}, \lambda_{1}=\lambda_{3} \lambda_{2}$ i.e. $\lambda=\lambda_{3}$ and $C g(x)+f(x)=0$, then $y=C$.

5) If $\mathrm{DE}(1)$ has QPS $y=y(x)$ with QP $\omega=c, \mathrm{QPC} \lambda=\frac{\lambda_{1}}{\lambda_{2}}, \lambda_{1}{ }^{2}=\lambda_{2} \lambda_{3}$, i.e. $\lambda^{2}=\frac{\lambda_{3}}{\lambda_{2}}$ and $C^{2} g(x)+C f(x)+h(x)=0$ then $y=C$.

Proof. Under the conditions of the theorem QPS for DE (1) and eq. (7) is also QPS to the equation

where

$$
g(x) y^{2}+p f(x) y+q h(x)=0 .
$$

$$
p=\frac{\lambda_{1}-1}{\lambda \lambda_{2}-1}, \quad q=\frac{1}{\lambda} \cdot \frac{\lambda_{3}-\lambda}{\lambda \lambda_{2}-1} .
$$

The last equation has solutions

$$
y=\frac{-p f(x) \pm \sqrt{p^{2} f^{2}(x)-4 q h(x) g(x)}}{2 g(x)},
$$

Since QPS to DE (1) is QPS for eq. (14), it satisfies the equation

$$
p \lambda\left(\lambda_{1}-\lambda \lambda_{2}\right) f(x) y+q\left(\lambda_{3}-\lambda^{2} \lambda_{2}\right) h(x)=0 .
$$

Thus we have:

1) If $\lambda \neq \frac{\lambda_{1}}{\lambda_{2}}, \lambda_{1}^{2}-\lambda_{2} \lambda_{3} \neq 0$ and $f(x) \neq 0$ then,

$$
y=\frac{\left(\lambda^{2} \lambda_{2}-\lambda_{3}\right) q}{\lambda\left(\lambda_{1}-\lambda \lambda_{2}\right) p} \cdot \frac{h(x)}{f(x)}=\mu_{2} \frac{h(x)}{f(x)},
$$

from where follows $\lambda=\frac{\lambda_{3}}{\lambda_{1}}, p=q, \mu_{2}=-1$ and $y=-\frac{h(x)}{f(x)}$. 
If we compare the obtained solution with the solution (16) or substituting it in the eq. (14) we have $h(x)=0$ and $y=0$.

2) If $\lambda \neq \frac{\lambda_{1}}{\lambda_{2}}, \lambda_{1}^{2}-\lambda_{2} \lambda_{3} \neq 0$ and $f(x)=0$ then we have the equation $g(x) y^{2}+q h(x)=0$ and $q\left(\lambda_{3}-\lambda^{2} \lambda_{2}\right) h(x)=0$ from where $h(x)=0$ and $y=0$.

3) If $\lambda=\frac{\lambda_{1}}{\lambda_{2}}$, (i.e. $p=1$ ) and $h(x)=0$ then QPS is $y=0$ or $y=C$ when $C g(x)+f(x)=0$.

4) If $\lambda=\frac{\lambda_{1}}{\lambda_{2}}$ and $\lambda=\lambda_{3}$, i.e. $\lambda_{1}=\lambda_{2} \lambda_{3}$, from where follows $p=1$ and $q=0$, then QPS is $y=0$ or $y=C$ if $C g(x)+f(x)=0$.

5) If $\lambda=\frac{\lambda_{1}}{\lambda_{2}}$ and $\lambda^{2}=\frac{\lambda_{3}}{\lambda_{2}}$, i.e. $\lambda_{1}^{2}=\lambda_{2} \lambda_{3}$, then $p=1, q=1$ and QPS is $y=0$ or $y=C$ if $C^{2} g(x)+C f(x)+h(x)=0$.

Remark 2.4. From eq. (14), after short transformations, we obtain that QPS for DE (1) is also a solution to the equation

$$
y^{\prime}+(1-p) f(x) y+(1-q) h(x)=0
$$

where $p$ and $q$ are gien by (15).

Under the conditions of the Theorem 2.6., solving eq. (18) as a linear DE, we can find all QPS for DE (1). They are given by the formula

$$
y=e^{-\alpha \gamma(x)}(C-\delta(x)),
$$

where $p=1-\alpha, q=1-\beta, \gamma(x)=\int f(x) d x, \delta(x)=\beta \int e^{\alpha \gamma(x)} h(x) d x$. In this case, from here we have also then only $y(x)=0$ or $y(x)=C$ are QPS for DE (1).

Remark 2.5. Some examples in [4] are special cases from the obtained results in this paper. 


\section{REFERENCES}

[1] J. Митевска, М. Кујумџиева Николоска, Квазипериодичност на решенијата на линеарна диференцијална равенка од I ред, Майемайички бил-

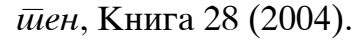

[2] J. Mitevska, M. Kujumdzieva Nikoloska, D. Dimitrovski, Quaziperiodicity of the solutions for linear nonhomogeneous differential equations of second order, Math. Macedonica, Vol. 2, 83-88 (2004).

[3] M. Kujumdzieva Nikoloska, J. Mitevska, Conditions of existence quasiperiodic solutions to Riccati differential equation, South-West University Journal, http:// press.swu.bg/epub/.

[4] Э. Камке, Справочник по обыкновенным дифференциальным уравнениям, Москва, 1971.

\section{Р е $з$ и м е \\ КВАЗИ-ПЕРИОДИЧНО РЕШЕНИЕ НА ДИФЕРЕНЦИЈАЛНАТА РАВЕНКА НА РИКАТИ}

Во овој труд даваме некои услови за постоење квазипериодични решенија на Рикатиевата диференцијална равенка и ги наоѓаме решенијата за случај на константен квази-период.

Клучни зборови: диференцијална равенка (DE); квази-период (QP); квазипериодично решение (QPS); квази-периодичен коефициент (QPC); равенка (еq.)

Adress:

Marija Kujumdžieva-Nikoloska

Faculty of Elecrical Engineering and Information Technologies,

"SS. Cyril and Methodius" University, Skopje,

P.O. Box 574, MK-1001 Skopje, Republic of Macedonia

marekn@feit.ukim.edu.mk

\section{Jordanka Mitevska}

Institute of Mathematics, Faculty of Natural Sciences and Mathematics,

"SS. Cyril and Methodius" University, Skopje,

P. O. Box 162, MK-1001 Skopje, Republic of Macedonia

jordankam@ukim.edu.mk

Received: 19. XII 2005

Accepted: 23. II 1006

Contributions, Sec. Math. Tech. Sci., XXVII-XXVIII, 1-2 (2006-2007), pp. 55-66 